\title{
COMPARISON OF SOME PURITIES, FLATNESSES AND INJECTIVITIES
}

\author{
WALID AL-KAWARIT AND FRANÇOIS COUCHOT
}

\begin{abstract}
In this paper, we compare $(n, m)$-purities for different pairs of positive integers $(n, m)$. When $R$ is a commutative ring, these purities are not equivalent if $R$ doesn't satisfy the following property: there exists a positive integer $p$ such that, for each maximal ideal $P$, every finitely generated ideal of $R_{P}$ is $p$-generated. When this property holds, then the $(n, m)$-purity and the $\left(n, m^{\prime}\right)$-purity are equivalent if $m$ and $m^{\prime}$ are integers $\geq n p$. These results are obtained by a generalization of Warfield's methods. There are also some interesting results when $R$ is a semiperfect strongly $\pi$-regular ring. We also compare $(n, m)$-flatnesses and $(n, m)$-injectivities for different pairs of positive integers $(n, m)$. In particular, if $R$ is right perfect and right self $\left(\aleph_{0}, 1\right)$-injective, then each $(1,1)$-flat right $R$-module is projective. In several cases, for each positive integer $p$, all $(n, p)$-flatnesses are equivalent. But there are some examples where the $(1, p)$-flatness is not equivalent to the $(1, p+1)$-flatness.
\end{abstract}

All rings in this paper are associative with unity, and all modules are unital. Let $n$ and $m$ be two positive integers. A right $R$-module $M$ is said to be $(n, m)$ presented if it is the factor module of a free right module of rank $n$ modulo a $m$-generated submodule. A short exact sequence $(\Sigma)$ of left $R$-modules is called $(n, m)$-pure if it remains exact when tensoring it with any $(n, m)$-presented right module. We say that $(\Sigma)$ is $\left(\aleph_{0}, m\right)$-pure exact (respectively $\left(n, \aleph_{0}\right)$-pure exact if, for each positive integer $n$ (respectively $m)(\Sigma)$ is $(n, m)$-pure exact. Let us observe that the $(1,1)$-pure exact sequences are exactly the RD-exact sequences (see [1]) and the $\left(\aleph_{0}, \aleph_{0}\right)$-exact sequences are the pure-exact sequences in the Cohn's sense. Similar results as in the classical theories of purity hold, with similar proofs. In particular, a left $R$-module is $(n, m)$-pure-projective if and only if it is a summand of a direct sum of $(m, n)$-presented left modules, and each left $R$-module has a $(n, m)$-pure-injective hull which is unique up to an isomorphism.

In this paper, we compare $(n, m)$-purities for different pairs of positive integers $(n, m)$. When $R$ is commutative, we shall see that some of these purities are equivalent only if $R$ satisfies the following property: there exists a positive integer $p$ such that, for each maximal ideal $P$, every finitely generated ideal of $R_{P}$ is $p$ generated. When this property holds, then $(n, m)$-purity and $\left(n, m^{\prime}\right)$-purity are equivalent if $m$ and $m^{\prime}$ are integers $\geq n p$. These results are obtained by using the following: if $R$ is a local commutative ring for which there exists a $(p+1)$-generated ideal, where $p$ is a positive integer, then, for each positive integer $n$, for each integer $m, n(p-1)+1 \leq m \leq n p+1$, there exists a $(n, m)$-presented $R$-module whose

2000 Mathematics Subject Classification. Primary 16D40, 16D50, 16D80.

Key words and phrases. $(n, m)$-pure submodule, $(n, m)$-flat module, $(n, m)$-injective module, $(n, m)$-coherent ring. 
endomorphism ring is local. It is a generalization of the Warfield's construction of indecomposable finitely presented modules when $R$ is not a valuation ring.

When $R$ is semiperfect and strongly $\pi$-regular, we show that there exists an integer $m>0$ such that, for any integer $n>0$, each $(n, m)$-pure exact sequence of right modules is $\left(n, \aleph_{0}\right)$-pure exact if and only if there exists an integer $p>0$ such that every finitely generated left ideal is $p$-generated.

As in [16 we define $(n, m)$-flat modules and $(n, m)$-injective modules. We also compare $(n, m)$-flatnesses and $(n, m)$-injectivities for different pairs of positive integers $(n, m)$. In particular, if $R$ is a right perfect ring which is right self $\left(\aleph_{0}, 1\right)$ injective, then each $(1,1)$-flat right $R$-module is projective. For many classes of rings, for each positive integer $p$, we show that the $(1, p)$-flatness implies the $\left(\aleph_{0}, p\right)$ flatness, but we have no general result. If $R$ is a local commutative ring with a non finitely generated maximal ideal $P$ satisfying $P^{2}=0$, then for each positive integer $p$, there exists an $R$-module which is $\left(\aleph_{0}, p\right)$-flat (resp, $\left(\aleph_{0}, p\right)$-injective) and which is not $(1, p+1)$-flat (resp, $(1, p+1)$-injective).

As in [16] we define left $(n, m)$-coherent rings. When $R$ is a commutative locally perfect ring which is $(1,1)$-coherent and self $(1,1)$-injective, we show that $R$ is an IF-ring, each $(1,1)$-flat $R$-module is flat and each $(1,1)$-injective $R$-module is FPinjective. For other classes of rings, for each positive integer $p$, we show that the left $(1, p)$-coherence implies the left $\left(\aleph_{0}, p\right)$-coherence, but we have no general result. If $R=V[[X]]$, the power series ring over a valuation domain $V$ whose order group is not isomorphic to $\mathbb{R}$, then $R$ is a $\left(\aleph_{0}, 1\right)$-coherent ring which is not $(1,2)$-coherent.

\section{1. $(n, m)$-PURE EXACT SEQUENCES}

By using a standard technique, (see for instance [4, Chapter I, Section 8]), we can prove the following theorem, and similar results hold if we replace $n$ or $m$ with $\aleph_{0}$.

Theorem 1.1. Assume that $R$ is an algebra over a commutative ring $S$ and $E$ is an injective $S$-cogenerator. Then, for each exact sequence $(\Sigma)$ of left $R$-modules $0 \rightarrow A \rightarrow B \rightarrow C \rightarrow 0$, the following conditions are equivalent:

(1) $(\Sigma)$ is $(n, m)$-pure;

(2) for each $(m, n)$-presented left module $G$ the sequence $\operatorname{Hom}_{R}(G,(\Sigma))$ is exact;

(3) every system of $n$ equations over $A$

$$
\sum_{j=1}^{m} r_{i, j} x_{j}=a_{i} \in A \quad(i=1, \ldots, n)
$$

with coefficients $r_{i, j} \in R$ and unknowns $x_{1}, \ldots, x_{m}$ has a solution in $A$ whenever it is solvable in $B$;

(4) the exact sequence of right $R$-modules $\operatorname{Hom}_{S}((\Sigma), E)$ is $(m, n)$-pure.

Propositions 1.2 and 1.3 can be deduced from [13, Theorem 1].

A left $R$-module $G$ is called $(n, m)$-pure-projective if for each $(n, m)$-pure exact sequence $0 \rightarrow A \rightarrow B \rightarrow C \rightarrow 0$ the sequence

$$
0 \rightarrow \operatorname{Hom}_{R}(G, A) \rightarrow \operatorname{Hom}_{R}(G, B) \rightarrow \operatorname{Hom}_{R}(G, C) \rightarrow 0
$$

is exact. Similar definitions can be given by replacing $n$ or $m$ by $\aleph_{0}$. From Theorem 1.1 and by using standard technique (see for instance 4, Chapter VI, Section 12]) we get the following proposition in which $n$ or $m$ can be replaced by $\aleph_{0}$ : 
Proposition 1.2. Let $G$ be a left $R$-module. Then the following assertions hold:

(1) there exists a $(n, m)$-pure exact sequence of left modules

$$
0 \rightarrow K \rightarrow F \rightarrow G \rightarrow 0
$$

where $F$ is a direct sum of $(m, n)$-presented left modules;

(2) $G$ is $(n, m)$-pure projective if and only if it is a summand of a direct sum of $(m, n)$-presented left modules.

A left $R$-module $G$ is called $(n, m)$-pure-injective if for each $(n, m)$-pure exact sequence $0 \rightarrow A \rightarrow B \rightarrow C \rightarrow 0$ the sequence

$$
0 \rightarrow \operatorname{Hom}_{R}(C, G) \rightarrow \operatorname{Hom}_{R}(B, G) \rightarrow \operatorname{Hom}_{R}(A, G) \rightarrow 0
$$

is exact.

If $M$ is a left module we put $M^{\sharp}=\operatorname{Hom}_{\mathbb{Z}}(M, \mathbb{Q} / \mathbb{Z})$. Thus $M^{\sharp}$ is a right module. It is the character module of $M$.

If $A$ is a submodule of a left $R$-module $B$, we say that $B$ is a $(n, m)$-pure essential extension of $A$ if $A$ is a $(n, m)$-pure submodule of $B$ and for each nonzero submodule $K$ of $B$ such that $A \cap K=0,(A+K) / K$ is not a $(n, m)$-pure submodule of $B / K$. If, in addition, $B$ is $(n, m)$-pure injective, we say that $B$ is a $(n, m)$-pure injective hull of $A$. In these above definitions and in the following proposition $n$ or $m$ can be replaced by $\aleph_{0}$.

Proposition 1.3. The following assertions hold:

(1) each left $R$-module is a $(n, m)$-pure submodule of a $(n, m)$-pure injective left module;

(2) each left $R$-module has a $(n, m)$-pure injective hull which is unique up to an isomorphism.

Proof. (1). Let $M$ be a left $R$-module. By Proposition 1.2 there exists a $(m, n)$ pure exact sequence of right $R$-modules $0 \rightarrow K \rightarrow F \rightarrow M^{\sharp} \rightarrow 0$ where $F$ is a direct sum of $(n, m)$-presented right modules. From Theorem 1.1 it follows that $\left(M^{\sharp}\right)^{\sharp}$ is a $(n, m)$-pure submodule of $F^{\sharp}$. By [3, Corollary 1.30] $M$ is isomorphic to a pure submodule of $\left(M^{\sharp}\right)^{\sharp}$. So, $M$ is isomorphic to a $(n, m)$-pure submodule of $F^{\sharp}$. By using the canonical isomorphism $\left(F \otimes_{R}-\right)^{\sharp} \cong \operatorname{Hom}_{R}\left(-, F^{\sharp}\right)$ we get that $F^{\sharp}$ is $(n, m)$-pure injective since $F$ is a direct sum of $(n, m)$-presented modules.

(2). Since (1) holds and every direct limit of $(n, m)$-pure exact sequences is $(n, m)$-pure exact too, we can adapt the method of Warfield's proof of existence of pure-injective hull to show (2)(see [11, Proposition 6]). We can also use [10, Proposition 4.5].

Proposition 1.4. Let $R$ be a commutative ring and let $(\Sigma)$ be a short exact sequence of $R$-modules. Then $(\Sigma)$ is $(n, m)$-pure if and only if, for each maximal ideal $P(\Sigma)_{P}$ is $(n, m)$-pure.

Proof. Assume that $(\Sigma)$ is $(n, m)$-pure and let $M$ be a $(n, m)$-presented $R_{P}$-module where $P$ is a maximal ideal. There exists a $(n, m)$-presented $R$-module $M^{\prime}$ such that $M \cong M_{P}^{\prime}$ and $M \otimes_{R_{P}}(\Sigma)_{P} \cong\left(M^{\prime} \otimes_{R}(\Sigma)\right)_{P}$. We deduce that $(\Sigma)_{P}$ is $(n, m)$-pure.

Conversely, suppose that $(\Sigma)$ is the sequence $0 \rightarrow A \rightarrow B \rightarrow C \rightarrow 0$. Let $M$ be a $(n, m)$-presented $R$-module. Then, for each maximal ideal $P,(\Sigma)_{P}$ is (n,m)-pure over $R$ since $M \otimes_{R}(\Sigma)_{P} \cong M_{P} \otimes_{R_{P}}(\Sigma)_{P}$. On the other hand, since $M \otimes_{R}\left(\prod_{P \in \operatorname{Max} R}(\Sigma)_{P}\right) \cong\left(\prod_{P \in \operatorname{Max} R} M \otimes_{R}(\Sigma)_{P}\right), \prod_{P \in \operatorname{Max} R} A_{P}$ is a $(n, m)$-pure 
submodule of $\prod_{P \in \operatorname{Max} R} B_{P}$. By [2, Lemme 1.3] $A$ is isomorphic to a pure submodule of $\prod_{P \in \operatorname{Max} R} A_{P}$. We successively deduce that $A$ is a $(n, m)$-pure submodule of $\prod_{P \in \operatorname{Max} R} B_{P}$ and $B$.

\section{COMPARISON OF PURITIES OVER A SEMIPERFECT RING}

In this section we shall compare $(n, m)$-purities for different pairs of integers $(n, m)$. In 7] some various purities are also compared. In particular some necessary and sufficient conditions on a ring $R$ are given for the (1,1)-purity to be equivalent to the $\left(\aleph_{0}, \aleph_{0}\right)$-purity.

The following lemma is due to Lawrence Levy, see [15, Lemma 1.3]. If $M$ be a finitely generated left (or right) $R$-module, we denote by gen $M$ its minimal number of generators.

Lemma 2.1. Let $R$ be a ring. Assume there exists a positive integer $p$ such that gen $A \leq p$ for each finitely generated left ideal $A$ of $R$. Then gen $N \leq p \times$ gen $M$, if $N$ is a finitely generated submodule of a finitely generated left $R$-module $M$.

From this lemma and Theorem 1.1 we deduce the following:

Proposition 2.2. Let $R$ be a ring. Assume there exists a positive integer $p$ such that gen $A \leq p$ for each finitely generated left ideal $A$ of $R$. Then, for each positive integer $n$ :

(1) each $(n, n p)$-pure exact sequence of right modules is $\left(n, \aleph_{0}\right)$-pure exact;

(2) each $(n p, n)$-pure exact sequence of left modules is $\left(\aleph_{0}, n\right)$-pure exact.

Corollary 2.3. Let $R$ be a left Artinian ring. Then there exists a positive integer $p$ such that, for each positive integer $n$ :

(1) each $(n, n p)$-pure exact sequence of right modules is $\left(n, \aleph_{0}\right)$-pure exact;

(2) each $(n p, n)$-pure exact sequence of left modules is $\left(\aleph_{0}, n\right)$-pure exact.

Proof. Each finitely generated left $R$-module $M$ has a finite length denoted by length $M$, and gen $M \leq$ length $M$. So, for each left ideal $A$ we have gen $A \leq$ length $R$. We choose $p=\sup \{$ gen $A \mid A$ left ideal of $R\}$ and we apply the previous proposition.

Let $R$ be a ring and $J$ its Jacobson radical. Recall that $R$ is semiperfect if $R / J$ is semisimple and idempotents lift modulo $J$.

Theorem 2.4. Let $R$ be semiperfect ring. Assume that each indecomposable finitely presented cyclic left $R$-module has a local endomorphism ring. The following conditions are equivalent:

(1) there exists an integer $p>0$ such that, for each integer $n>0$, each $(n, n p)$ pure exact sequence of right modules is $\left(n, \aleph_{0}\right)$-pure exact;

(2) there exists an integer $p>0$ such that, for each integer $n>0$, each $(n p, n)$ pure exact sequence of left modules is $\left(\aleph_{0}, n\right)$-pure exact;

(3) there exists an integer $q>0$ such that each $(1, q)$-pure exact sequence of right modules is $\left(1, \aleph_{0}\right)$-pure exact;

(4) there exists an integer $q>0$ such that each $(q, 1)$-pure exact sequence of left modules is $\left(\aleph_{0}, 1\right)$-pure exact;

(5) there exists an integer $q>0$ such that each indecomposable finitely presented cyclic left module is q-related; 
(6) there exists an integer $p>0$ such that gen $A \leq p$ for each finitely generated left ideal $A$ of $R$.

Moreover, if each indecomposable finitely presented left $R$-module has a local endomorphism ring, these conditions are equivalent to the following:

(7) there exist two positive integers $n, m$ such that each $(n, m)$-pure exact sequence of right modules is $\left(n, \aleph_{0}\right)$-pure exact;

(8) there exist two positive integers $n, m$ such that each $(m, n)$-pure exact sequence of left modules is $\left(\aleph_{0}, n\right)$-pure exact;

Proof. By Proposition $2.2(6) \Rightarrow(1)$. By Theorem $1.1(1) \Leftrightarrow(2),(3) \Leftrightarrow(4)$ and $(7) \Leftrightarrow(8)$. It is obvious that $(2) \Rightarrow(4)$ and $(2) \Rightarrow(7)$.

$(4) \Rightarrow(5)$. Let $C$ be an indecomposable finitely presented cyclic left module. Then $C$ is $(q, 1)$-pure-projective. So, $C$ is a direct summand of a finite direct sum of $(1, q)$-presented left modules. Since $R$ is semiperfect, we may assume that these $(1, q)$-presented left modules are indecomposable. So, by Krull-Schmidt theorem $C$ is $(1, q)$-presented.

$(5) \Rightarrow(6)$. Let $A$ be a finitely generated left ideal. Then $R / A=\oplus_{i=1}^{t} R / A_{i}$ where, for each $i=1, \ldots, t, A_{i}$ is a left ideal and $R / A_{i}$ is indecomposable. We have the following commutative diagram with exact horizontal sequences:

$$
\begin{aligned}
& 0 \rightarrow \oplus_{i=1}^{t} A_{i} \rightarrow R^{t} \rightarrow \oplus_{i=1}^{t} R / A_{i} \rightarrow 0 \\
& 0 \rightarrow \quad A \quad \rightarrow \quad R \quad \rightarrow \quad R / A \quad \rightarrow \quad 0
\end{aligned}
$$

Since the right vertical map is an isomorphism, we deduce from the snake lemma that the other two vertical homomorphisms have isomorphic cokernels. It follows that gen $A \leq t q+1$ because gen $A_{i} \leq q$ by (5). On the other hand, let $P$ be a projective cover of $R / A$. Then $P$ is isomorphic to a direct summand of $R$. We know that the left module $R$ is a finite direct sum of indecomposable projective modules. Let $s$ the number of these indecomposable summands. It is easy to show that $t \leq s$. So, if $p=s q+1$, then gen $A \leq p$.

$(8) \Rightarrow(5)$. Let $C$ be an indecomposable finitely presented cyclic left module. Then $C$ is $(m, n)$-pure-projective. So, $C$ is a direct summand of a finite direct sum of $(n, m)$-presented left modules. Since $R$ is semiperfect, we may assume that these $(n, m)$-presented left modules are indecomposable. So, by the Krull-Schmidt theorem $C$ is $(1, m)$-presented.

A ring $R$ is said to be strongly $\pi$-regular if, for each $r \in R$, there exist $s \in R$ and an integer $q \geq 1$ such that $r^{q}=r^{q+1} s$. By [3, Theorem 3.16] each strongly $\pi$-regular $R$ satisfies the following condition: for each $r \in R$, there exist $s \in R$ and an integer $q \geq 1$ such that $r^{q}=s r^{q+1}$. Recall that a left $R$-module $M$ is said to be Fitting if for each endomorphism $f$ of $M$ there exists a positive integer $t$ such that $M=\operatorname{ker} f^{t} \oplus f^{t}(M)$.

Lemma 2.5. Let $R$ be a strongly $\pi$-regular semiperfect ring. Then:

(1) each finitely presented cyclic left (or right) R-module is Fitting;

(2) each indecomposable finitely presented cyclic left (or right) $R$-module has a local endomorphism ring.

Proof. In [3, Lemma 3.21] it is proven that every finitely presented $R$-module is a Fitting module if $R$ is a semiperfect ring with $\mathrm{M}_{n}(R)$ strongly $\pi$-regular for all $n$. We do a similar proof to show (1). 
(2). By [3, Lemma 2.21] each indecomposable Fitting module has a local endomorphism ring.

Corollary 2.6. Let $R$ be a strongly $\pi$-regular semiperfect ring. The following conditions are equivalent:

(1) there exists an integer $p>0$ such that, for each integer $n>0$, each $(n, n p)$ pure exact sequence of right modules is $\left(n, \aleph_{0}\right)$-pure exact;

(2) there exists an integer $p>0$ such that, for each integer $n>0$, each $(n p, n)$ pure exact sequence of left modules is $\left(\aleph_{0}, n\right)$-pure exact;

(3) there exists an integer $q>0$ such that each $(1, q)$-pure exact sequence of right modules is $\left(1, \aleph_{0}\right)$-pure exact;

(4) there exists an integer $q>0$ such that each $(q, 1)$-pure exact sequence of left modules is $\left(\aleph_{0}, 1\right)$-pure exact;

(5) there exists an integer $q>0$ such that each indecomposable finitely presented cyclic left module is q-related;

(6) there exists an integer $p>0$ such that gen $A \leq p$ for each finitely generated left ideal $A$ of $R$.

Moreover, if $\mathrm{M}_{n}(R)$ is strongly $\pi$-regular for all $n>0$, these conditions are equivalent to the following:

(7) there exist two positive integers $n, m$ such that each $(n, m)$-pure exact sequence of right modules is $\left(n, \aleph_{0}\right)$-pure exact;

(8) there exist two positive integers $n, m$ such that each $(m, n)$-pure exact sequence of left modules is $\left(\aleph_{0}, n\right)$-pure exact;

Proof. By Lemma 2.5 each indecomposable finitely presented cyclic left $R$-module has a local endomorphism ring. If $\mathrm{M}_{n}(R)$ is strongly $\pi$-regular for all $n$, then by [3. Lemmas 3.21 and 2.21] each indecomposable finitely presented left $R$-module has a local endomorphism ring. So, we apply Theorem 2.4 .

Recall that a ring $R$ is right perfect if each flat right $R$-module is projective.

Corollary 2.7. Let $R$ be a right perfect ring. The following conditions are equivalent:

(1) there exists an integer $p>0$ such that, for each integer $n>0$, each $(n, n p)$ pure exact sequence of right modules is $\left(n, \aleph_{0}\right)$-pure exact;

(2) there exists an integer $p>0$ such that, for each integer $n>0$, each $(n p, n)$ pure exact sequence of left modules is $\left(\aleph_{0}, n\right)$-pure exact;

(3) there exists an integer $q>0$ such that each $(1, q)$-pure exact sequence of right modules is $\left(1, \aleph_{0}\right)$-pure exact;

(4) there exist two positive integers $n, m$ such that each $(n, m)$-pure exact sequence of right modules is $\left(n, \aleph_{0}\right)$-pure exact;

(5) there exist two positive integers $n, m$ such that each $(m, n)$-pure exact sequence of left modules is $\left(\aleph_{0}, n\right)$-pure exact;

(6) there exists an integer $p>0$ such that gen $A \leq p$ for each finitely generated left ideal $A$ of $R$.

Proof. For all $n>0, \mathrm{M}_{n}(R)$ is right perfect. Since each right perfect ring satisfies the descending chain condition on finitely generated left ideals, then $\mathrm{M}_{n}(R)$ is strongly $\pi$-regular for all $n>0$. We apply Corollary 2.6. 


\section{COMPARISON OF PURITIES OVER A COMMUTATIVE RING}

In the sequel of this section $R$ is a commutative local ring, except in Theorem 3.5 We denote respectively by $P$ and $k$ its maximal ideal and its residue field

Let $M$ be a finitely presented $R$-module. Recall that gen $M=\operatorname{dim}_{k} M / P M$. Let $F_{0}$ be a free $R$-module whose rank is gen $M$ and let $\phi: F_{0} \rightarrow M$ be an epimorphism. Then ker $\phi \subseteq P F_{0}$. We put rel $M=$ gen ker $\phi$. Let $F_{1}$ be a free $R$-module whose rank is rel $M$ and let $f: F_{1} \rightarrow F_{0}$ be a homomorphism such that $\operatorname{im} f=\operatorname{ker} \phi$. Then ker $f \subseteq P F_{1}$. For any $R$-module $N$, we put $N^{*}=\operatorname{Hom}_{R}(N, R)$. Let $f^{*}: F_{0}^{*} \rightarrow F_{1}^{*}$ be the homomorphism deduced from $f$. We set $\mathrm{D}(M)=$ coker $f^{*}$ the Auslander and Bridger's dual of $M$. The following proposition is the version in commutative case of [14, Theorem 2.4]:

Proposition 3.1. Assume that $M$ has no projective summand. Then:

(1) ker $f^{*} \subseteq P F_{0}^{*}$ and im $f^{*} \subseteq P F_{1}^{*}$;

(2) $M \cong \mathrm{D}(\mathrm{D}(M))$ and $\mathrm{D}(M)$ has no projective summand;

(3) gen $\mathrm{D}(M)=\operatorname{rel} M$ and $\operatorname{rel} \mathrm{D}(M)=$ gen $M$;

(4) if $M=M_{1} \oplus M_{2}$ then gen $M=$ gen $M_{1}+$ gen $M_{2}$ and rel $M=\operatorname{rel} M_{1}+\operatorname{rel} M_{2}$.

(5) $\operatorname{End}_{R}(\mathrm{D}(M))$ is local if and only if so is $\operatorname{End}_{R}(M)$.

Lemma 3.2. Let $M$ be a finitely generated $R$-module, $s$ an endomorphism of $M$ and $\bar{s}$ the endomorphism of $M / P M$ induced by $s$. Then $s$ is an isomorphism if and only if so is $\bar{s}$.

Proof. If $s$ is an isomorphism it is obvious that so is $\bar{s}$. Conversely, coker $s=0$ by Nakayama lemma. So, $s$ is surjective. By using a Vasconcelos's result (see [4, Theorem V.2.3]) $s$ is bijective.

Proposition 3.3. Assume that there exists an ideal $A$ with gen $A=p+1$ where $p$ is a positive integer. Then, for each positive integers $n$ and $m$ with $(n-1) p+1 \leq$ $m \leq n p+1$, there exists a finitely presented $R$-module $W_{p, n, m}$ whose endomorphism ring is local and such that gen $W_{p, n, m}=n$ and rel $W_{p, n, m}=m$.

Proof. Suppose that $A$ is generated by $a_{1}, \ldots, a_{p}, a_{p+1}$. Let $F$ be a free module of rank $n$ with basis $e_{1}, \ldots, e_{n}$ and let $K$ be the submodule of $F$ generated by $x_{1}, \ldots, x_{m}$ where these elements are defined in the following way: if $j=p q+r$ where $1 \leq r \leq p, x_{j}=a_{r} e_{q+1}$ if $r \neq 1$ or $q=0$, and $x_{j}=a_{p+1} e_{q}+a_{1} e_{q+1}$ else; when $m=p n+1, x_{m}=a_{p+1} e_{n}$. We put $W_{p, n, m}=F / K$. We can say that $W_{p, n, m}$ is named by the following $n \times m$ matrix, where $r=m-p(n-1)$ :

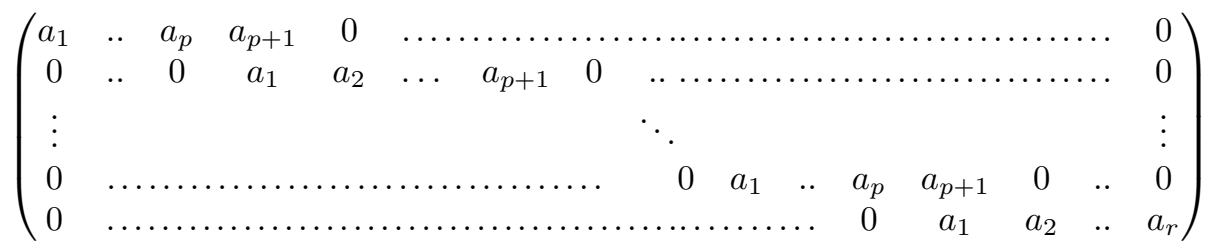

Since $K \subseteq P F$, gen $W_{p, n, m}=n$. Now we consider the following relation: $\sum_{j=0}^{m} c_{j} x_{j}=0$. From the definition of the $x_{j}$ we get the following equality:

$$
\sum_{q=0}^{n-2}\left(\sum_{i=1}^{p+1} c_{p q+i} a_{i}\right) e_{q+1}+\left(\sum_{i=1}^{r} c_{p(n-1)+i} a_{i}\right) e_{n}=0 .
$$


Since $\left\{e_{1}, \ldots, e_{n}\right\}$ is a basis and gen $A=p+1$ we deduce that $c_{j} \in P, \forall j, 1 \leq j \leq m$. So, rel $W_{p, n, m}=m$.

Let $s \in \operatorname{End}_{R}\left(W_{p, n, m}\right)$. Then $s$ is induced by an endomorphism $\tilde{s}$ of $F$ which satisfies $\tilde{s}(K) \subseteq K$. For each $j, 1 \leq j \leq n$, there exists a family $\left(\alpha_{i, j}\right)$ of elements of $R$ such that:

$$
\tilde{s}\left(e_{j}\right)=\sum_{i=1}^{n} \alpha_{i, j} e_{i}
$$

Since $\tilde{s}(K) \subseteq K, \forall j, 1 \leq j \leq m, \exists$ a family $\left(\beta_{i, j}\right)$ of elements of $R$ such that:

$$
\tilde{s}\left(x_{j}\right)=\sum_{i=1}^{m} \beta_{i, j} x_{i}
$$

From (11), (2) and the equality $x_{1}=a_{1} e_{1}$ if follows that:

$$
\sum_{q=1}^{n} \alpha_{q, 1} a_{1} e_{q}=\sum_{q=0}^{n-2}\left(\sum_{i=1}^{p+1} \beta_{p q+i, 1} a_{i}\right) e_{q+1}+\left(\sum_{i=1}^{r} \beta_{p(n-1)+i, 1} a_{i}\right) e_{n} .
$$

Then, we get:

$$
\begin{gathered}
\forall q, 1 \leq q \leq n-1, \quad \alpha_{q, 1} a_{1}=\sum_{i=1}^{p+1} \beta_{p(q-1)+i, 1} a_{i} \\
\text { and } \quad \alpha_{n, 1} a_{1}=\sum_{i=1}^{r} \beta_{p(n-1)+i, 1} a_{i} .
\end{gathered}
$$

We deduce that: $\forall q, 2 \leq q \leq n, \beta_{p(q-2)+p+1,1} \in P$ and $\beta_{p(q-1)+1,1} \equiv \alpha_{q, 1}[P]$. So,

$$
\forall q, 2 \leq q \leq n, \alpha_{q, 1} \in P .
$$

Now, let $j=p \ell+1$ where $1 \leq \ell \leq(n-1)$. In this case, $x_{j}=a_{p+1} e_{\ell}+a_{1} e_{\ell+1}$. From (1) and (2) it follows that:

$$
\sum_{q=1}^{n}\left(\alpha_{q, \ell} a_{p+1}+\alpha_{q, \ell+1} a_{1}\right) e_{q}=\sum_{q=0}^{n-2}\left(\sum_{i=1}^{p+1} \beta_{p q+i, j} a_{i}\right) e_{q+1}+\left(\sum_{i=1}^{r} \beta_{p(n-1)+i, j} a_{i}\right) e_{n} .
$$

Then, we get:

$$
\begin{gathered}
\forall q, 1 \leq q \leq n-1, \quad \alpha_{q, \ell} a_{p+1}+\alpha_{q, \ell+1} a_{1}=\sum_{i=1}^{p+1} \beta_{p(q-1)+i, j} a_{i} \\
\quad \text { and } \quad \alpha_{n, \ell} a_{p+1}+\alpha_{n, \ell+1} a_{1}=\sum_{i=1}^{r} \beta_{p(n-1)+i, j} a_{i} .
\end{gathered}
$$

We deduce that

$$
\forall q, \ell, 1 \leq q, \ell \leq(n-1), \alpha_{q, \ell} \equiv \beta_{p(q-1)+p+1, j}[P] \text { and } \alpha_{q+1, \ell+1} \equiv \beta_{p q+1, j}[P],
$$

whence $\alpha_{q, \ell} \equiv \alpha_{q+1, \ell+1}[P]$. Consequently, $\forall q, 1 \leq q \leq n, \alpha_{q, q} \equiv \alpha_{1,1}[P]$ and $\forall t, 1 \leq t \leq(n-1), \forall q, 1 \leq q \leq(n-t), \alpha_{q+t, q} \equiv \alpha_{1+t, 1} \equiv 0[P]$ by (3). Let $\bar{s}$ be the endomorphism of $W_{p, n, m} / P W_{p, n, m}$ induced by $s$. If $\alpha_{1,1}$ is a unit then $\bar{s}$ is an isomorphism, else $\overline{\mathbf{1}_{W_{p, n, m}}-s}$ is an isomorphism. By Lemma 3.2 we conclude that either $s$ or $\left(\mathbf{1}_{W_{p, n, m}}-s\right)$ is an isomorphism. Hence, $\operatorname{End}_{R}\left(W_{p, n, m}\right)$ is local. 
Remark 3.4. Observe that $\mathrm{D}\left(W_{1, n-1, n}\right)$ is isomorphic to the indecomposable module built in the proof of [12, Theorem 2].

Theorem 3.5. Let $R$ be a commutative ring. The following assertions hold:

(1) Assume that, for any integer $p>0$, there exists a maximal ideal $P$ and a finitely generated ideal $A$ of $R_{P}$ such that $\operatorname{gen}_{R_{P}} A \geq p+1$. Then, if $(n, m)$ and $(r, s)$ are two different pairs of integers, the $(n, m)$-purity and the $(r, s)$-purity are not equivalent.

(2) Assume that, there exists an integer $p>0$ such that, for each maximal ideal $P$, for any finitely generated ideal $A$ of $R_{P}$, $\operatorname{gen}_{R_{P}} A \leq p$. Then:

(a) for each integer $n>0$ the $\left(\aleph_{0}, n\right)$-purity (respectively $\left(n, \aleph_{0}\right)$-purity) is equivalent to the $(n p, n)$-purity (respectively ( $n, n p)$-purity);

(b) if $p>1$, then, for each integer $n>0$, for each integer $m, 1 \leq m \leq$ $n(p-1)$, the ( $n, m)$-purity (respectively $(m, n)$-purity) is not equivalent to the $(n, m+1)$-purity (respectively $(m+1, n)$-purity).

Proof. By Proposition 1.4 we may assume that $R$ is local with maximal $P$. By Theorem 1.1 the $(n, m)$-purity and the $(r, s)$-purity are equivalent if and only if so are the $(m, n)$-purity and the $(s, r)$-purity.

(1). Suppose that $r>n$ and let $t=\min (m, s)$. Let $q$ be the greatest divisor of $(r-1)$ which is $\leq t$ and $p=(r-1) / q$. Let $A$ be a finitely generated ideal such that gen $A>p$. By way of contradiction, suppose that $W_{p, q, r}$ is $(n, m)$ pure-projective. By Proposition $1.2 W_{p, q, r}$ is a summand of $\oplus_{i \in I} F_{i}$ where $I$ is a finite set and $\forall i \in I, F_{i}$ is a $(m, n)$-presented $R$-module. Since its endomorphism ring is local, $W_{p, q, r}$ is an exchange module (see [3, Theorem 2.8]). So, we have $W_{p, q, r} \oplus\left(\oplus_{i \in I} G_{i}\right) \cong\left(\oplus_{i \in I} H_{i}\right) \oplus\left(\oplus_{i \in I} G_{i}\right)$ where $\forall i \in I, G_{i}$ and $H_{i}$ are submodules of $F_{i}$ and $F_{i}=G_{i} \oplus H_{i}$. Let $G=\oplus_{i \in I} G_{i}$. Then $G$ is finitely generated. By [4, Proposition V.7.1] $\operatorname{End}_{R}(G)$ is semilocal. By using Evans's theorem ([3, Corollary 4.6]) we deduce that $W_{p, q, r} \cong\left(\oplus_{i \in I} H_{i}\right)$. Since $W_{p, q, r}$ is indecomposable, we get that it is $(m, n)$-presented. This contradicts that rel $W_{p, q, r}=r>n$.

(2)(a) is an immediate consequence of Proposition 2.2.

(2)(b). There exist two integers $q, t$ such that $m+1=(q-1)(p-1)+t$ with $n \geq q \geq 1$ and $1 \leq t \leq p$. As in (1) we prove that $W_{p-1, q, m+1}$ is not $(m, n)$-pureprojective.

Remark 3.6. In the previous theorem, when there exists an integer $p>1$ such that, for any finitely generated ideal $A$ gen $A \leq p$, we don't know if the $(n, m)$-purity and the $(n, m+1)$-purity are equivalent when $n(p-1)+1 \leq m \leq n p-1$. If $R$ is a local ring with maximal $P$ with residue field $k$ such that $P^{2}=0$ and $\operatorname{dim}_{k} P=p$ it is easy to show that each finitely presented $R$-module $F$ with gen $F=n$ and rel $F=n p$ is semisimple. So, the $(n p, n)$-purity is equivalent to the $(n p-1, n)$-purity.

\section{4. $(n, m)$-FLAT MOdules AND $(n, m)$-INJECTIVE MOduleS}

Let $M$ be a right $R$-module. We say that $M$ is $(n, m)$-flat if for any $m$-generated submodule $K$ of a $n$-generated free left $R$-module $F$, the natural map: $M \otimes_{R} K \rightarrow$ $M \otimes_{R} F$ is a monomorphism. We say that $M$ is $\left(\aleph_{0}, m\right)$-flat (respectively $\left(n, \aleph_{0}\right)$ flat) if $M$ is $(n, m)$-flat for each integer $n>0$ (respectively $m>0$ ). We say that $M$ is $(n, m)$-injective if for any $m$-generated submodule $K$ of a $n$-generated free right $R$-module $F$, the natural map: $\operatorname{Hom}_{R}(F, M) \rightarrow \operatorname{Hom}_{R}(K, M)$ is an epimorphism. 
We say that $M$ is $\left(\aleph_{0}, m\right)$-injective (respectively $\left(n, \aleph_{0}\right)$-injective) if $M$ is $(n, m)$ injective for each integer $n>0$ (respectively $m>0$ ). A ring $R$ is called left self $(n, m)$-injective if $R$ is $(n, m)$-injective as left $R$-module.

If $R$ is a commutative domain, then an $R$-module is $(1,1)$-flat (respectively $(1,1)$ injective) if and only if it is torsion-free (respectively divisible).

The following propositions can be proved with standard technique: see 16, Theorem 4.3 and Proposition 2.3]. In these propositions the integers $n$ or $m$ can be replaced with $\aleph_{0}$.

Proposition 4.1. Assume that $R$ is an algebra over a commutative ring $S$ and let $E$ be an injective $S$-cogenerator. Let $M$ be a right $R$-module. The following conditions are equivalent:

(1) $M$ is $(n, m)$-flat;

(2) each exact sequence $0 \rightarrow L \rightarrow N \rightarrow M \rightarrow 0$ is $(n, m)$-pure, where $L$ and $N$ are right $R$-modules;

(3) for each $(m, n)$-presented right module $F$, every homomorphism $f: F \rightarrow M$ factors through a free right $R$-module;

(4) $\operatorname{Hom}_{S}(M, E)$ is a $(n, m)$-injective left $R$-module.

Proposition 4.2. Let $M$ be a right module. The following conditions are equivalent:

(1) $M$ is $(n, m)$-injective;

(2) each exact sequence $0 \rightarrow M \rightarrow L \rightarrow N \rightarrow 0$ is $(m, n)$-pure, where $L$ and $N$ are right $R$-modules;

(3) $M$ is a $(m, n)$-pure submodule of its injective hull.

Proposition 4.3. Let $R$ be a commutative ring. Then an $R$-module $M$ is $(n, m)$ flat if and only if, for each maximal ideal $P, M_{P}$ is $(n, m)$-flat over $R_{P}$.

Lemma 4.4. Let $M$ be p-generated right $R$-module where $p$ is a positive integer. Then $M$ is flat if and only if it is $(1, p)$-flat.

Proof. Only "if" requires a proof. Let $A$ be a left ideal. Assume that $M$ is generated by $x_{1}, \ldots, x_{p}$. So, if $z \in M \otimes_{R} A, z=\sum_{i=1}^{p} x_{i} \otimes a_{i}$ where $a_{1}, \ldots, a_{p} \in A$. Suppose that the image of $z$ in $M \otimes_{R} R$ is 0 . If $A^{\prime}$ is the left ideal generated by $a_{1}, \ldots, a_{p}$, if $z^{\prime}$ is the element of $M \otimes_{R} A^{\prime}$ defined by $z^{\prime}=\sum_{i=1}^{p} x_{i} \otimes a_{i}$, then $z$ (respectively 0 ) is the image of $z^{\prime}$ in $M \otimes_{R} A$ (respectively $M \otimes_{R} R$ ). Since $M$ is $(1, p)$-flat we successively deduce that $z^{\prime}=0$ and $z=0$.

It is well known that each $\left(1, \aleph_{0}\right)$-flat right module is $\left(\aleph_{0}, \aleph_{0}\right)$-flat. For each positive integer $p$, is each $(1, p)$-flat right module $\left(\aleph_{0}, p\right)$-flat?

The following theorem and Theorem 4.11 give a partial answer to this question.

Theorem 4.5. Let $p$ be a positive integer and let $R$ be a ring. For each positive integer $n$, assume that, for each p-generated submodule $G$ of the left $R$-module $R^{n} \oplus R,\left(G \cap R^{n}\right)$ is the direct limit of its p-generated submodules. Then a right $R$-module $M$ is $(1, p)$-flat if and only if it is $\left(\aleph_{0}, p\right)$-flat.

Proof. We shall prove that $M$ is $(n, p)$-flat by induction on $n$. Let $G$ be a $p$ generated submodule of the left $R$-module $R^{n+1}=R^{n} \oplus R$. Let $\pi$ be the projection of $R^{n+1}$ onto $R$ and $G^{\prime}=\pi(G)$. Then $G^{\prime}$ is a $p$-generated left module. We put 
$H=G \cap R^{n}$. We have the following commutative diagram with exact horizontal sequences:

$$
\begin{aligned}
& M \otimes_{R} H \quad \rightarrow \quad M \otimes_{R} G \quad \stackrel{1_{M} \otimes \pi}{\longrightarrow} M \otimes_{R} G^{\prime} \quad \rightarrow \quad 0 \\
& \downarrow \quad \downarrow \quad \downarrow \\
& 0 \rightarrow M \otimes_{R} R^{n} \rightarrow M \otimes_{R} R^{n+1} \stackrel{1_{M} \otimes \pi}{\longrightarrow} M \otimes_{R} R \rightarrow 0
\end{aligned}
$$

Let $u: G \rightarrow R^{n+1}, u^{\prime}: G^{\prime} \rightarrow R, w: R^{n} \rightarrow R^{n+1}$ be the inclusion maps and let $v=\left.u\right|_{H}$. Then $\left(1_{M} \otimes u^{\prime}\right)$ is injective. Let $H^{\prime}$ be a $p$-generated submodule of $H$. By the induction hypothesis $M$ is $(n, p)$-flat. So, $\left(1_{M} \otimes\left(\left.v\right|_{H^{\prime}}\right)\right)$ is injective. It follows that $\left(1_{M} \otimes v\right)$ is injective too. We conclude that $\left(1_{M} \otimes u\right)$ is injective and $M$ is $\left(\aleph_{0}, p\right)$-flat.

Corollary 4.6. Let $p$ be a positive integer and let $R$ be a ring such that each left ideal is $(1, p)$-flat. Then, for each positive integer $q \leq p$, a right $R$-module $M$ is $(1, q)$-flat if and only if it is $\left(\aleph_{0}, q\right)$-flat.

Proof. Let the notations be as in the previous theorem. Since $G^{\prime}$ is a flat left $R$ module by Lemma 4.4, $H$ is a pure submodule of $G$. Let $\left\{g_{1}, \ldots, g_{q}\right\}$ be a spanning set of $G$ and let $h_{1}, \ldots, h_{t} \in H$. For each $k, 1 \leq k \leq t$ there exist $a_{k, 1}, \ldots, a_{k, q} \in R$ such that $h_{k}=\sum_{i=1}^{q} a_{k, i} g_{i}$. It follows that there exist $g_{1}^{\prime}, \ldots, g_{q}^{\prime} \in H$ such that $\forall k, 1 \leq k \leq t, h_{k}=\sum_{i=1}^{q} a_{k, i} g_{i}^{\prime}$. So, each finitely generated submodule of $H$ is contained in a $q$-generated submodule. We conclude by applying Theorem 4.5.

Corollary 4.7. Let $R$ be a commutative local ring with maximal P. Assume that $P^{2}=0$. Let $q$ a positive integer. Then:

(1) each $(1, q)$-flat module is $\left(\aleph_{0}, q\right)$-flat;

(2) each $(1, q)$-injective module is $\left(\aleph_{0}, q\right)$-injective.

Proof. Let the notations be as in the previous theorem. We may assume that $G \subseteq P R^{n+1}$. Then $G$ is a semisimple module and $H$ is a direct summand of $G$. So, (1) is a consequence of Theorem 4.5 .

(2). Let $M$ be a $(1, q)$-injective module. We shall prove by induction on $n$ that $M$ is $(n, q)$-injective. We have the following commutative diagram:

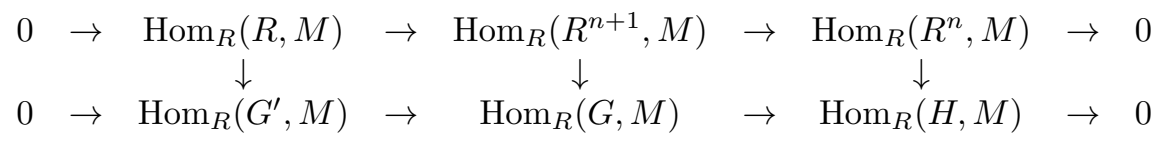

where the horizontal sequences are exact. By the induction hypothesis the left and the right vertical maps are surjective. It follows that the middle vertical map is surjective too.

By [9, Example 5.2] or [6, Theorem 2.3], for each integer $n>0$, there exists a $\operatorname{ring} R$ for which each finitely generated left ideal is $(1, n)$-flat (hence $\left(\aleph_{0}, n\right)$-flat by Corollary 4.6) but there is a finitely generated left ideal which is not $(1, n+1)$-flat. The following proposition gives other examples in the commutative case.

Proposition 4.8. Let $R$ be a commutative local ring with maximal ideal $P$ and residue field $k$. Assume that $P^{2}=0$ and $\operatorname{dim}_{k} P>1$. Then, for each positive integer $p<\operatorname{dim}_{k} P$, there exists:

(1) a $(p+1,1)$-presented $R$-module which is $\left(\aleph_{0}, p\right)$-flat but not $(1, p+1)$-flat;

(2) a $\left(\aleph_{0}, p\right)$-injective $R$-module which is not $(1, p+1)$-injective. 
Proof. (1). Let $F$ be a free $R$-module of rank $(p+1)$ with basis $\left\{e_{1}, \ldots, e_{p}, e_{p+1}\right\}$, let $\left(a_{1}, \ldots, a_{p}, a_{p+1}\right)$ be a family of linearly independent elements of $P$, let $K$ be the submodule of $F$ generated by $\sum_{i=1}^{p+1} a_{i} e_{i}$ and let $M=F / K$. Then $M \cong D\left(W_{p, 1, p+1}\right)$ (see the proof of Proposition 3.3). First, we show that $K$ is a $(1, p)$-pure submodule of $F$. We consider the following equation:

$$
\sum_{j=1}^{p} r_{j} x_{j}=s\left(\sum_{i=1}^{p+1} a_{i} e_{i}\right)
$$

where $r_{1}, \ldots, r_{p}, s \in R$ and with unknowns $x_{1}, \ldots, x_{p}$. Assume that this equation has a solution in $F$. Suppose there exists $\ell, 1 \leq \ell \leq p$, such that $r_{\ell}$ is a unit. For each $j, 1 \leq j \leq p$, we put $x_{j}^{\prime}=\delta_{j, \ell} r_{\ell}^{-1} s\left(\sum_{i=1}^{p+1} a_{i} e_{i}\right)$. It is easy to check that $\left(x_{1}^{\prime}, \ldots, x_{p}^{\prime}\right)$ is a solution of (4) in $K$. Now we assume that $r_{j} \in P, \forall j, 1 \leq j \leq p$. Suppose that $\left(x_{1}, \ldots, x_{p}\right)$ is a solution of (4) in $F$. For each $j, 1 \leq j \leq p, x_{j}=$ $\sum_{i=1}^{p+1} c_{j, i} e_{i}$, where $c_{j, i} \in R$. We get the following equality:

$$
\sum_{i=1}^{p+1}\left(\sum_{j=1}^{p} r_{j} c_{j, i}\right) e_{i}=\sum_{i=1}^{p+1} s a_{i} e_{i}
$$

We deduce that:

$$
\forall i, \quad 1 \leq i \leq p+1, \quad \sum_{j=1}^{p} r_{j} c_{j, i}=s a_{i}
$$

So, if $s$ is a unit, $\forall i, 1 \leq i \leq p+1, a_{i} \in \sum_{j=1}^{p} R r_{j}$. It follows that

$$
\operatorname{dim}_{k}\left(\sum_{i=1}^{p+1} R a_{i}\right) \leq p
$$

that is false. So, $s \in P$. In this case (4) has the nil solution. Hence $M$ is $\left(\aleph_{0}, p\right)$-flat by Proposition 4.1(2) and Corollary 4.7

By way of contradiction suppose that $M$ is $(1, p+1)$-flat. It follows that $K$ is a $(1, p+1)$-pure submodule of $F$ by Proposition 4.1. Since $M$ is $(1, p+1)$-pureprojective we deduce that $M$ is free. This is false.

(2). Let $E$ be an injective $R$-cogenerator. Then $\operatorname{Hom}_{R}(M, E)$ is $\left(\aleph_{0}, p\right)$-injective but not $(1, p+1)$-injective by Proposition 4.1(4).

In a similar way we show the following proposition.

Proposition 4.9. Let $R$ be a commutative local ring with maximal ideal $P$. Assume that $P^{2}=0$. Let $M$ be a $(m, 1)$-presented $R$-module with $m>1$, let $\left\{x_{1}, \ldots, x_{m}\right\}$ be a spanning set of $M$ and let $\sum_{j=1}^{m} a_{j} x_{j}=0$ be the relation of $M$, where $a_{1}, \ldots, a_{m} \in$ $P$. If $p=\operatorname{gen}\left(\sum_{j=1}^{m} R a_{j}\right)-1>0$, then:

(1) $M$ is $\left(\aleph_{0}, p\right)$-flat but not $(1, m)$-flat;

(2) $\operatorname{Hom}_{R}(M, E)$ is $\left(\aleph_{0}, p\right)$-injective but not $(1, m)$-injective, where $E$ is an injective $R$-cogenerator.

When $R$ is an arithmetical commutative ring, i.e. its lattice of ideals is distributive, each $(1,1)$-flat module is flat and by [4, Theorem VI.9.10] the converse holds if $R$ is a commutative domain (it is also true if each principal ideal is flat). However we shall see that there exist non-arithmetical commutative rings for which each 
$(1,1)$-flat module is flat. Recall that a left (or right) $R$-module $M$ is torsionless if the natural map $M \rightarrow\left(M^{*}\right)^{*}$ is injective.

Proposition 4.10. For each ring $R$ the following conditions are equivalent:

(1) $R$ is right self $\left(\aleph_{0}, 1\right)$-injective;

(2) each finitely presented cyclic left $R$-module is torsionless;

(3) each finitely generated left ideal $A$ satisfies $A=1-\operatorname{ann}(\mathrm{r}-\operatorname{ann}(A))$.

Proof. We prove $(1) \Leftrightarrow(2)$ as [5, Theorem 2.3] and (2) $\Leftrightarrow(3)$ is easy.

Theorem 4.11. Let $R$ be a right perfect ring which is right self $\left(\aleph_{0}, 1\right)$-injective. Then each $(1,1)$-flat right module is projective.

Proof. Let $M$ be a $(1,1)$-flat right $R$-module. It is enough to show that $M$ is flat. Let $A$ be a finitely generated left ideal of $R$. Assume that $\left\{a_{1}, \ldots, a_{n}\right\}$ is a minimal system of generators of $A$ with $n>1$. Let $z \in M \otimes_{R} A$ such that its image in $M$ is 0 . We have $z=\sum_{i=1}^{n} y_{i} \otimes a_{i}$, where $y_{1}, \ldots, y_{n} \in M$, and $\sum_{i=1}^{n} y_{i} a_{i}=0$. For each $i, 1 \leq i \leq n$, we set $A_{i}=\sum_{\substack{j=1 \\ j \neq i}}^{n} R a_{j}$. Then, $\forall i, 1 \leq i \leq n$, $A_{i} \subset A$. For each finitely generated left ideal $B$ we have $B=1-\operatorname{ann}(\mathrm{r}-\operatorname{ann}(B))$. It follows that, $\forall i, 1 \leq i \leq n, \mathrm{r}-\operatorname{ann}(A) \subset \mathrm{r}-\operatorname{ann}\left(A_{i}\right)$. Let $\left.b_{i} \in \mathrm{r}-\operatorname{ann}\left(A_{i}\right)\right) \backslash$ $\mathrm{r}-\operatorname{ann}(A)$. Then $y_{i} a_{i} b_{i}=0$. From the $(1,1)$-flatness of $M$ we deduce that $y_{i}=$ $\sum_{k=1}^{m_{i}} y_{i, k}^{\prime} c_{i, k}$, where $y_{i, 1}^{\prime}, \ldots, y_{i, m_{i}}^{\prime} \in M$ and $c_{i, 1}, \ldots, c_{i, m_{i}} \in R$ with $c_{i, k} a_{i} b_{i}=$ $0, \forall k, 1 \leq k \leq m_{i}$. It follows that $z=\sum_{i=1}^{n}\left(\sum_{k=1}^{m_{i}} y_{i, k}^{\prime} \otimes c_{i, k} a_{i}\right)$. Let $A^{(1]}$ be the left ideal generated by $\left\{c_{i, k} a_{i} \mid 1 \leq i \leq n, 1 \leq k \leq m_{i}\right\}$. Then $A^{(1)} \subset A$; else, $\forall i, 1 \leq i \leq n, a_{i}=\sum_{j=1}^{n}\left(\sum_{k=1}^{m_{j}} d_{i, j, k} c_{j, k} a_{j}\right)$ with $d_{i, j, k} \in R$; we get that $a_{i} b_{i}=\sum_{j=1}^{n}\left(\sum_{k=1}^{m_{j}} d_{i, j, k} c_{j, k} a_{j} b_{i}\right)$; but $a_{j} b_{i}=0$ if $j \neq i$ and $c_{i, k} a_{i} b_{i}=0$; so, there is a contradiction because the second member of the previous equality is 0 while $a_{i} b_{i} \neq 0$. Let $\left\{a_{1}^{(1)}, \ldots, a_{n_{1}}^{(1)}\right\}$ be a minimal system of generators of $A^{(1)}$. So, $z=\sum_{i=1}^{n_{1}} y_{i}^{(1)} \otimes a_{i}^{(1)}$ where $y_{1}^{(1)}, \ldots, y_{n_{1}}^{(1)} \in M$, and $z$ is the image of $z^{(1)} \in M \otimes_{R} A^{(1)}$ defined by $z^{(1)}=\sum_{i=1}^{n_{1}} y_{i}^{(1)} \otimes a_{i}^{(1)}$. If $n_{1} \leq 1$ we conclude that $z^{(1)}=0$ since $M$ is (1,1)-flat, and $z=0$. If $n_{1}>1$, in the same way we get that $z^{(1)}$ is the image of an element $z^{(2)} \in M \otimes_{R} A^{(2)}$ where $A^{(2)}$ is a left ideal such that $A^{(2)} \subset A^{(1)}$. If gen $A^{(2)}>1$ we repeat this process, possibly several times, until we get a left ideal $A^{(l)}$ with gen $A^{(l)} \leq 1$; this is possible because $R$ satisfies the descending chain condition on finitely generated left ideals since it is right perfect (see 8, Théorème 5 p.130]). The (1,1)-flatness of $M$ implies that $z^{(l)}=0$ and $z=0$. So, $M$ is projective.

Let $\mathcal{P}$ be a ring property. We say that a commutative ring $R$ is locally $\mathcal{P}$ if $R_{P}$ satisfies $\mathcal{P}$ for each maximal ideal $P$.

The following corollary is a consequence of Theorem 4.11 and Proposition 4.3

Corollary 4.12. Let $R$ be a commutative ring which is locally perfect and locally self $\left(\aleph_{0}, 1\right)$-injective. Then each $(1,1)$-flat $R$-module is flat.

\section{5. $(n, m)$-COHERENT RINGS}

We say that a ring $R$ is left $(n, m)$-coherent if each $m$-generated submodule of a $n$-generated free left $R$-module is finitely presented. We say that $R$ is left $\left(\aleph_{0}, m\right)$ coherent (respectively $\left(n, \aleph_{0}\right)$-coherent) if for each integer $n>0$ (respectively $m>$ $0) R$ is left $(n, m)$-coherent. The following theorem can be proven with standard 
technique: see [16, Theorems 5.1 and 5.7]. In this theorem the integers $n$ or $m$ can be replaced with $\aleph_{0}$.

Theorem 5.1. Let $R$ be a ring and $n, m$ two fixed positive integers. Assume that $R$ is an algebra over a commutative ring $S$. Let $E$ be an injective $S$-cogenerator. Then the following conditions are equivalent:

(1) $R$ is left $(n, m)$-coherent;

(2) any direct product of right $(n, m)$-flat $R$-modules is $(n, m)$-flat;

(3) for any set $\Lambda, R^{\Lambda}$ is a $(n, m)$-flat right $R$-module;

(4) any direct limit of a direct system of $(n, m)$-injective left $R$-modules is $(n, m)$-injective;

(5) for any exact sequence of left modules $0 \rightarrow A \rightarrow B \rightarrow C \rightarrow 0, C$ is $(n, m)$ injective if so is $B$ and if $A$ is a $\left(\aleph_{0}, m\right)$-pure submodule of $B$;

(6) for each $(n, m)$-injective left $R$-module $M, \operatorname{Hom}_{S}(M, E)$ is $(n, m)$-flat.

It is well known that each left $\left(1, \aleph_{0}\right)$-coherent ring is left $\left(\aleph_{0}, \aleph_{0}\right)$-coherent. For each positive integer $p$, is each left $(1, p)$-coherent ring left $\left(\aleph_{0}, p\right)$ coherent?

Propositions 5.2 and 5.3 and Theorem 5.4 give a partial answer to this question.

Proposition 5.2. Let $p$ be a positive integer and let $R$ be a ring. For each positive integer $n$, assume that, for each p-generated submodule $G$ of the left $R$-module $R^{n} \oplus R,\left(G \cap R^{n}\right)$ is the direct limit of its p-generated submodules. Then the following conditions are equivalent:

(1) $R$ is left $(1, p)$-coherent;

(2) $R$ is left $\left(\aleph_{0}, p\right)$-coherent.

Moreover, when these conditions hold each $(1, p)$-injective left module is $\left(\aleph_{0}, p\right)$ injective.

Proof. It is obvious that $(2) \Rightarrow(1)$.

$(1) \Rightarrow(2)$. Let $\Lambda$ be a set. By Theorem $5.1 R^{\Lambda}$ is a $(1, p)$-flat right module. From Theorem 4.5 we deduce that $R^{\Lambda}$ is a $\left(\aleph_{0}, p\right)$-flat right module. By using again Theorem 5.1 we get (2).

Let $M$ be a $(1, p)$-injective left module. By Theorem $5.1 M^{\sharp}$ is a $(1, p)$-flat right $R$-module. Then it is also $\left(\aleph_{0}, p\right)$-flat. We deduce that $\left(M^{\sharp}\right)^{\sharp}$ is a $\left(\aleph_{0}, p\right)$-injective left module. Since $M$ is a pure submodule of $\left(M^{\sharp}\right)^{\sharp}$, it follows that $M$ is $\left(\aleph_{0}, p\right)$ injective too.

Proposition 5.3. Let $R$ be a commutative perfect ring. Then $R$ is Artinian if and only if it is $(1,1)$-coherent.

Proof. Suppose that $R$ is $(1,1)$-coherent. Since $R$ is perfect, $R$ is a finite product of local rings. So, we may assume that $R$ is local with maximal $P$. Let $S$ be a minimal non-zero ideal of $R$ generated by $s$. Then $P$ is the annihilator of $s$. So, $P$ is finitely generated and it is the sole prime ideal of $R$. Since all prime ideals of $R$ are finitely generated, $R$ is Noetherian. On the other hand $R$ satisfies the descending chain condition on finitely generated ideals. We conclude that $R$ is Artinian.

Except in some particular cases, we don't know if each $(1, p)$-injective module is $\left(\aleph_{0}, p\right)$-injective, even if we replace $p$ by $\aleph_{0}$. 
Theorem 5.4. Let $R$ be a ring which is right perfect, left $(1,1)$-coherent and right self $\left(\aleph_{0}, 1\right)$-injective. Then each $(1,1)$-injective left module is $\left(\aleph_{0}, \aleph_{0}\right)$-injective and $R$ is left coherent.

Proof. Let $M$ be a left $(1,1)$-injective module. By Theorem $5.1 M^{\sharp}$ is $(1,1)$-flat. Whence $M^{\sharp}$ is projective by Theorem 4.11. We do as in the proof of Proposition 5.2 to conclude that $M$ is $\left(\aleph_{0}, \aleph_{0}\right)$-injective.

For each set $\Lambda, R^{\Lambda}$ is a $(1,1)$-flat right module by Theorem 5.1, It follows that $R^{\Lambda}$ is a projective right module by Theorem 4.11 .

Recall that a ring is quasi-Frobenius if it is Artinian and self-injective.

Corollary 5.5. Let $R$ be a quasi-Frobenius ring. Then, for each right (or left) $R$-module $M$, the following conditions are equivalent:

(1) $M$ is (1,1)-flat;

(2) $M$ is projective;

(3) $M$ is injective;

(4) $M$ is (1,1)-injective.

Proof. It is well known that $(2) \Leftrightarrow(3)$. By Theorem $4.11(1) \Leftrightarrow(2)$ because $R$ satisfies the conditions of this theorem, and it is obvious that $(3) \Rightarrow(4)$ and the converse holds by Theorem 5.4 .

We prove the following theorem as [2, Théorème 1.4].

Theorem 5.6. Let $R$ be a commutative ring and $n, m$ two fixed positive integers. The following conditions are equivalent:

(1) $R$ is $(n, m)$-coherent;

(2) for each multiplicative subset $S$ of $R, S^{-1} R$ is $(n, m)$-coherent, and for each $(n, m)$-injective $R$-module $M, S^{-1} M$ is $(n, m)$-injective over $S^{-1} R$;

(3) For each maximal ideal $P, R_{P}$ is $(n, m)$-coherent and for each $(n, m)$ injective $R$-module $M, M_{P}$ is $(n, m)$-injective over $R_{P}$.

Recall that a ring $R$ is a right $I F$-ring if each right injective $R$-module is flat.

Theorem 5.7. Let $R$ be a commutative ring which is locally perfect, $(1,1)$-coherent and self $(1,1)$-injective. Then:

(1) $R$ is coherent, self $\left(\aleph_{0}, \aleph_{0}\right)$-injective and locally quasi-Frobenius;

(2) each $(1,1)$-flat module is flat;

(3) each $(1,1)$-injective module is $\left(\aleph_{0}, \aleph_{0}\right)$-injective.

Proof. By Theorem 5.6 $R_{P}$ is $(1,1)$-coherent and $(1,1)$-injective for each maximal ideal $P$. Let $a$ be a generator of a minimal non-zero ideal of $R_{P}$. Then $P R_{P}$ is the annihilator of $a$ and consequently $P R_{P}$ is finitely generated over $R_{P}$. Since all prime ideals of $R_{P}$ are finitely generated, we deduce that $R_{P}$ is Artinian for each maximal ideal $P$. Moreover, the $(1,1)$-injectivity of $R_{P}$ implies that the socle of $R_{P}$ (the sum of all minimal non-zero ideals) is simple. It follows that $R_{P}$ is quasi-Frobenius for each maximal ideal $P$.

Let $M$ be a $\left(\aleph_{0}, \aleph_{0}\right)$-injective $R$-module. By Theorem $5.6 M_{P}$ is $(1,1)$-injective for each maximal ideal $P$. By Corollary $5.5 M_{P}$ is injective for each maximal ideal $P$. We conclude that $R$ is self $\left(\aleph_{0}, \aleph_{0}\right)$-injective and it is coherent by Theorem 5.6

If $M$ is $(1,1)$-injective, we prove as above that $M_{P}$ is injective for each maximal ideal $P$. It follows that $M$ is $\left(\aleph_{0}, \aleph_{0}\right)$-injective. 
The second assertion is an immediate consequence of Corollary 4.12

The following proposition is easy to prove:

Proposition 5.8. A ring $R$ is left $\left(\aleph_{0}, 1\right)$-coherent if and only if each finitely generated right ideal has a finitely generated left annihilator.

Example 5.9. Let $V$ be a non-Noetherian (commutative) valuation domain whose order group is not the additive group of real numbers and let $R=V[[X]]$ be the power series ring in one indeterminate over $V$. Since $R$ is a domain, $R$ is $\left(\aleph_{0}, 1\right)$ coherent. But, in [1] it is proven that there exist two elements $f$ and $g$ of $R$ such that $R f \cap R g$ is not finitely generated. By using the exact sequence $0 \rightarrow R f \cap R g \rightarrow$ $R f \oplus R g \rightarrow R f+R g \rightarrow 0$ we get that $R f+R g$ is not finitely presented. So, $R$ is not $(1,2)$-coherent.

\section{REFERENCES}

[1] Anderson, M. and Watkins, J. ((1987)). Coherence of power series rings over pseudo-Bezout domains. J. Algebra, 107:187-194.

[2] Couchot, F. ((1982)). Exemples d'anneaux auto fp-injectifs. Comm. Algebra, 10(4):339-360.

[3] Facchini, A. ((1998)). Module theory. Endomorphism rings and direct sum decompositions in some classes of modules. Progress in Mathematics (Boston, Mass.). Birkhäuser, Basel.

[4] Fuchs, L. and Salce, L. ((2001)). Modules over Non-Noetherian Domains. Number 84 in Mathematical Surveys and Monographs. American Mathematical Society, Providence.

[5] Jain, S. ((1973)). Flatness and FP-injectivity. Proc. Amer. Math. Soc., 41(2):437-442.

[6] Jøndrup, S. ((1971)). p.p.rings and finitely generated flat ideals. Proc. Amer. Math. Soc., 28:431-435.

[7] Puninski, G., Prest, M., and Rothmaler, P. ((1999)). Rings described by various purities. Comm. Algebra, 27(5):2127-2162.

[8] Renault, G. ((1975)). Algèbre non commutative. Collection "Varia Mathematica". GauthierVillars.

[9] Shamsuddin, A. ((2001)). $n$-injective and $n$-flat modules. Comm. Algebra, 29(5):2039-2050.

[10] Stenström, B. ((1967)). Pure submodules. Ark. Mat., 7:159-171.

[11] Warfield, R. ((1969)a). Purity and algebraic compactness for modules. Pac. J. Math., 28(3):689-719.

[12] Warfield, R. ((1970)). Decomposability of finitely presented modules. Proc. Amer. Math. Soc., 25:167-172.

[13] Warfield, R. B. ((1969)b). Relatively injective modules. unpublished paper.

[14] Warfield, R. ((1975)). Serial Rings and Finitely Presented Modules. J. Algebra, 37(2):187-222.

[15] Wiegand, R. and Wiegand, S. ((1975)). Finitely generated modules over Bezout rings. Pac. J. Math., 58(2):655-664.

[16] Zhang, X., Chen, J., and Zhang, J. ((2005)). On ( $m, n)$-injective modules and $(m, n)$-coherent rings. Algebra Colloq., 12(1):149-160.

Université de Caen Basse-Normandie, CNRS UMr 6139 LMNO, F-14032 Caen, France

E-mail address: walid.al-kawarit@unicaen.fr

Université de Caen Basse-Normandie, CNRS UMR 6139 LMNO, F-14032 Caen, France

E-mail address: francois.couchot@unicaen.fr 